\title{
Area Delay Modeling Based on Traffic Assignment
}

\author{
Lingyun Zhu and Feng Chen \\ Information Science and Technology, University of Science and Technology of China, Hefei, Anhui, China, 230027 \\ *Corresponding author
}

\begin{abstract}
Delay model is the basis for traffic flow optimal control. The current work mainly focuses on delay model of intersection, and less research is paid to area delay model. To meet the demands of area coordination control for urban traffic network, this paper proposes an area delay modeling method based on traffic assignment. In this work, traffic flow of OD demands is assigned for each path respectively. The average delay of each path is calculated according to the regularities of external entrance and internal entrance delay. And then the area delay can be obtained. A typical area consisting of four intersections is employed to validate the proposed method. The results indicate that the model results coincide well with the simulation results
\end{abstract}

Keywords-traffic assignment; area delay model; OD; simulation

\section{INTRODUCTION}

Area delay is an important index to evaluate urban transportation service and operating efficiency. It reflects not only time cost of travelers, but also the operating state of transportation system. The area operations is in good condition, the delay is low and vice versa. For the complexity of vehicle running and closely correlation among intersections, the area delay cannot obtain based on single intersection. The current studies of urban traffic delay model mainly focus on the intersection and artery, less research has been paid on area delay model [1]. Area delay model is the basis of traffic flow analysis and area coordination control. Therefore, it is necessary to analyze and model area delay.

In this paper, according to the OD matrix, traffic assignment is used to figure out the traffic flow of each path, then delay of each path is calculated, finally we can obtain area delay.

This paper is organized as follows: the basic principle and solving method of traffic assignment model are introduced, then a method of area delay model is given, finally, a simulation experiment and analysis is carried out based on a typical area consisting of four intersections.

\section{TRAFFIC ASSIGNMENT MODEL}

The most basic criterion of traffic assignment model is the Wardorp [2] Equilibrium Criterion, which includes: the User equilibrium (UE) criterion and the System Optimal (SO) Criterion. UE model assumes that travelers are able to master all information of whole road network, while SO model is premised on the basis that all travelers obey scheduling of one organization, but these are difficult to achieve in real world [3].

In this paper, the Stochastic User Equilibrium (SUE) based traffic assignment model is introduced, which considers that travelers are only aware of local road network information. A discrete choice model based on path selection behavior is established. The path selection probability of the model is given by $P_{p}^{r s}=e^{-\theta u_{p}^{r s}} / \sum_{l} e^{-\theta u_{l}^{r s}}$ whose equivalent extreme value model [3] is as follows.

$$
\begin{gathered}
\min z(x)=\frac{1}{\theta} \sum_{r, s, p} f_{p}^{r s} \ln f_{p}^{r s}+\sum_{a} \int_{0}^{x_{a}} t_{a}(x) d x \\
\text { s.t. } \quad \sum_{p} f_{p}^{r s}=q_{r s}, \quad \forall r, s \\
f_{p}^{r s} \geq 0, \quad \forall r, s, p \\
x_{a}=\sum_{r, s, p} f_{p}^{r s} \delta_{a, p}^{r s}, \quad \forall a
\end{gathered}
$$

where, $z(x)$ is the objective function, $x_{a}$ is traffic volume of directed link $a, t_{a}(x)$ represents the impedance of link $a, f_{p}^{\mathrm{rs}}$ and $u_{p}^{r s}$ respectively represent the vehicle flow and impedance of path $p$ about OD pairs $(r, s), q_{r s}$ represents the OD flow from origin point $r$ to destination $s . \delta_{a, p}^{r s}$ stands for correlation coefficient of the link and path, and its value is 1 if link $a$ is on path $p$ between OD pairs $(r, s)$, otherwise $\delta_{a, p}^{r s}=0$.

The equation (1) is a nonlinear programming problem, which can be solved by using method of successive weighted averages (MSWA). The concrete solution steps are as follows [4].

Step0. Obtain a set of effective paths between each OD;

Step1. Initialize each link flow as 0 , according to the impedance of each link, the path impedance is calculated; then the initial flow rate of each path is obtained according to the path selection probability; finally let the iteration number $n$ be 1 ;

Step2. Get link flow $x_{a}^{n}$ according to path flow $f_{p}^{r s(n)}$, then update link impedance and path impedance. 
Step3. Calculate additional path flow $g_{p}^{r s(n)}$ with new path impedance and path selection probability.

Step4. Update path flow:

$$
\begin{aligned}
f_{p}^{r s(n+1)} & =f_{p}^{r s(n)}+\chi^{(n)}\left(g_{p}^{r s(n)}-f_{p}^{r s(n)}\right) \\
\chi^{(n)} & =\frac{n^{2}}{1^{2}+2^{2}+3^{2}+\ldots+n^{2}}
\end{aligned}
$$

Step5. If the error between $g_{p}^{r s(n)}$ and $f_{p}^{r s(n)}$ is less than a certain threshold, end this cycle, else go to Step2.

\section{AREA DELAY MODELING}

Area delay is generated by vehicles running through corresponding local network. It can be transformed to the weighted average of path delay and path flow.

$$
d=\frac{\sum_{r, s, p} f_{p}^{\mathrm{rs}} d_{p}}{\sum_{r, s, p} f_{p}^{\mathrm{rs}}}
$$

where, $d$ denotes average area delay of each vehicle, $d_{p}$ is average path delay of each vehicle.

In this paper, delay model is obtained in term of the following assumptions:

1. The cycle of all intersections in area is consistent, because offset optimization is based on the same cycle of intersections.

2. In the local road network, road traffic volume is no more than road traffic capacity.

3. The influence of queue length on delay is ignored. In area, trajectory of vehicle cannot form loop.

The path delay is divided into two parts:

1. External entrance delay. The delay is caused by external traffic flow accessing the area. Traffic flow state is regarded as a random arrival state.

2. Internal entrance delay. The delay is generated by internal traffic flow in the area. The adjacent intersections in road network are very close. It may lead to vehicle queuing overflow.

Path delay is composed of an external entrance delay and a number of internal entrance delays:

$$
d_{p}=d_{\text {out }}+\sum_{i=1}^{N} d_{i n, i}
$$

where, $d_{\text {out }}$ and $d_{i n, i}$ represent external entrance delay and internal entrance delay respectively.

The traffic flow of external entrance arrive randomly, vehicle delay can be calculated in term of Webster Formula [6]:

$$
d_{\text {out }}=\frac{T\left(1-\lambda_{\text {out }}\right)^{2}}{2\left(1-s_{\text {out }} \lambda_{\text {out }}\right)}+\frac{s_{\text {out }}{ }^{2}}{2 q_{\text {out }}\left(1-s_{\text {out }}\right)}
$$

where, $s_{\text {out }}, \lambda_{\text {out }}, q_{\text {out }}$ represent saturation of external entrance, split and average vehicle arrived rate respectively. $T$ is the intersection cycle..

The internal entrance delay is divided into two parts, which is caused by a motorcade accessing from the upstream intersection to the downstream intersection, the first part delay is the head of motorcade blocked at the downstream intersection when traffic light is red; the second part delay is the tail of motorcade blocked at the downstream intersection when traffic light turns red. The delay model is as the follows:

$$
d_{\text {in }}=\frac{\alpha \cdot d_{\text {head }}+(1-\alpha) \cdot d_{\text {tail }}}{n_{\text {down }}}
$$

$$
d_{\text {head }}=\frac{q_{\text {down }} u_{\text {down }}\left\{\Delta t-\left[\frac{l_{\text {up, down }}}{v}\right] \bmod (T)\right\}^{2}}{2\left(u_{\text {down }}-q_{\text {down }}\right)}
$$

$$
\begin{aligned}
& d_{\text {tail }}=q_{\text {down }} T\left(1-\lambda_{\text {down }}\right)\left\{\left[\frac{l_{\text {up down }}}{v}\right] \bmod (T)-\Delta t\right\} \\
& -\frac{1}{2} q_{\text {down }}\left\{\left[\frac{l_{\text {up down }}}{v}\right] \bmod (T)-\Delta t\right\}^{2} \\
& +\frac{1}{2} q_{\text {down }}^{2}\left\{\left[\frac{l_{\text {up down }}}{v}\right] \bmod (T)-\Delta t\right\}^{2} / u_{\text {down }}
\end{aligned}
$$

$$
\begin{gathered}
\alpha=\left\{\begin{array}{l}
0,\left[\frac{l_{u p, \text { down }}}{v}\right] \bmod (T)>\Delta t \\
1,\left[\frac{l_{\text {up down }}}{v}\right] \bmod (T) \leq \Delta t
\end{array}\right. \\
n_{\text {down }}=T \cdot q_{\text {down }}
\end{gathered}
$$

$$
\Delta t=\phi_{\text {up }, \text { down }}
$$

where, $d_{\text {head }}$ is the delay which is caused by the head of 
motorcade blocked in one intersection cycle, $d_{\text {tail }}$ is the delay which is caused by the tail of motorcade blocked in one intersection cycle, $q_{\text {down }}, u_{\text {down }}$ respectively represent traffic flow and traffic capacity of the downstream intersection. $l_{\text {up,down }} / v$ is the time that motorcade has taken from upstream to downstream. The head of motorcade is blocked when $\alpha=1$, otherwise, the tail of motorcade is blocked. $n_{\text {down }}$ represents the count of vehicles which run through the downstream in one cycle. $\Delta t$ represents the start time difference that motorcade run through the upstream intersection and downstream intersection. $\phi_{u p, d o w n}$ is the offset between the upstream intersection and downstream intersection.

Equation (5) is suitable for offset optimization of arterial coordination [7]. In the arterial coordination, motorcade runs through the upstream and downstream intersection along the artery. Both upstream and downstream intersection execute coordinate phase, so we can use offset $\phi_{u p, d o w n}$ to represent $\Delta t$. But in the area, the phase may be different when the motorcade passes the two intersections, it is necessary to modify the original equation.

As shown in Figure 1, $s_{u p}$ and $s_{\text {down }}$ represent upstream intersection and downstream intersection, $T$ is the public cycle, $s_{u p}$ has $n$ phases, the splits are: $\lambda_{u p, 1}, \ldots, \lambda_{u p, n} ; s_{\text {down }}$ has $m$ phases, the splits are: $\lambda_{\text {down }, 1}, \ldots, \lambda_{\text {down }, m}$, the offset is $\phi_{\text {up }, \text { down }}$, we have:

$$
\begin{aligned}
& \varphi_{i, j}=\left(\sum_{k=i}^{n} T \cdot \lambda_{u p, k}+\sum_{k=1}^{j-1} T \cdot \lambda_{\text {down }, k}+\varphi_{\text {up }, \text { down }}\right) \bmod (T) \\
& 1 \leq i \leq n, 1 \leq j \leq m
\end{aligned}
$$

where, $\varphi_{i, j}$ represents delay time of phase $i$ with respect to phase $j, \lambda_{u p, k}$ and $\lambda_{\text {down }, k}$ represents the split of kth phase of $s_{u p}$ and $s_{\text {down }}$ respectively.

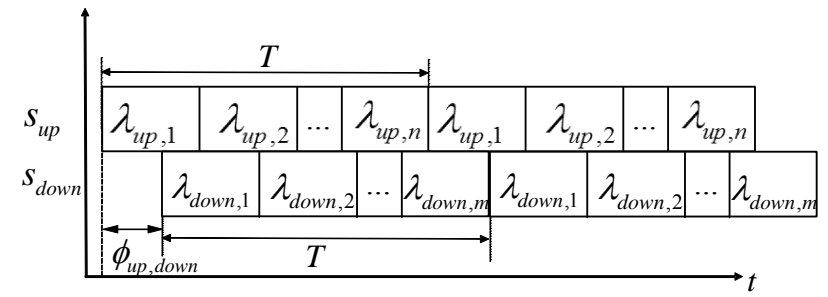

FIGURE I. THE PHASE INTERVAL TIME BETWEEN UPSTREAM AND DOWNSTREAM INTERACTION

Equation (15) is modified:

$$
\Delta t=\varphi_{u p_{i}, \text { down }_{j}}
$$

where, $u p_{i}$ represents that phase $i$ is executed when motorcade runs through $s_{u p}$, down $n_{j}$ denotes that phase $i$ is executed when motorcade runs through $s_{\text {down }}$.

In conclusion, the solution procedure of area delay is as follows:

Step1. According to $(1) \sim(4)$ to get traffic flow $f_{p}^{\text {rs }}$ of each path.

Step2. Calculate external entrance delay and internal entrance delay of each path in term of $(4) \sim(7)$, then get path delay using (3).

Step3. Figure out the area delays with (7).

\section{Simulation Results AND ANALysis}

In order to validate the model proposed in this paper, microscopic traffic simulation software is used, which is developed by laboratory of the data Fusion \& intelligent traffic system, University of Science and Technology of China. A typical area consisting of the four intersections is selected, each intersection has four phases, the cycle is 120 seconds, and there is twenty-four sections, fifty-six OD pairs, 104 valid paths in the road network Traffic capacity of each lane is $1500 \mathrm{veh} / \mathrm{h}$.

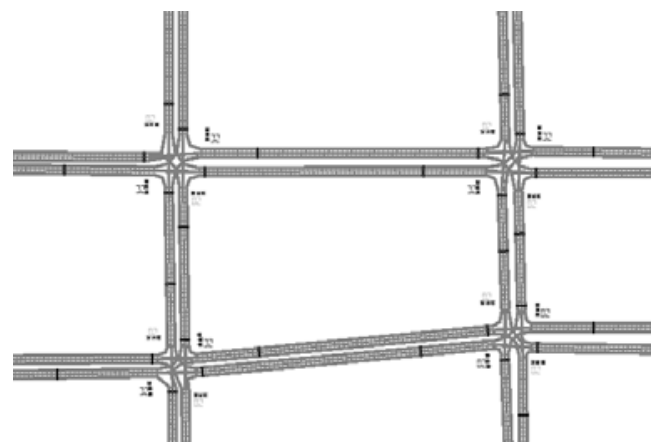

FIGURE II. AN AREA OF FOUR INTERSECTION

The area delay under different OD demands is computed in the experiment, the results are shown as follows:

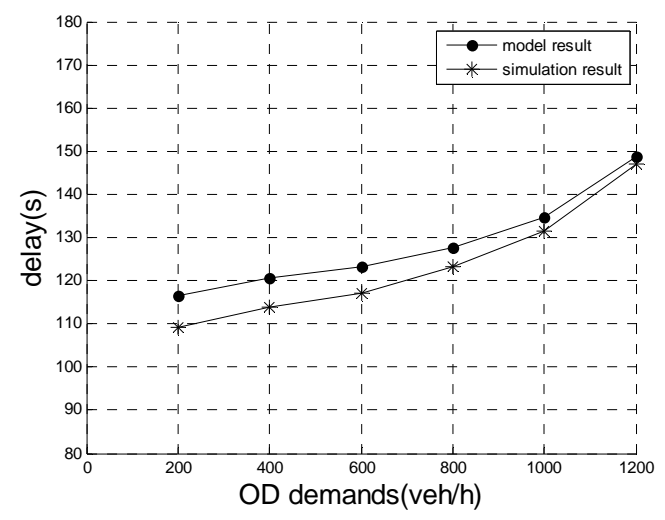

FIGURE III. LINE CHART OF THEORY AND SIMULATION DATA

TABLE I. ERROR BETWEEN THEORETICAL SIMULATION

\begin{tabular}{|c|c|c|c|c|c|c|}
\hline OD demand(veh/h) & $\mathbf{2 0 0}$ & $\mathbf{4 0 0}$ & $\mathbf{6 0 0}$ & $\mathbf{8 0 0}$ & $\mathbf{1 0 0 0}$ & $\mathbf{1 2 0 0}$ \\
\hline Error(s) & 7.6 & 6.8 & 6.4 & 5.2 & 3.3 & 1.7 \\
\hline
\end{tabular}


As seen in the figure 3 and table 1, the traffic flow is dispersed corresponding to low traffic flow rate. For random arrival of vehicles, there has a deviation between theoretical results and simulation data. The motorcades traffic flow is formed when traffic flow rate is high this proposed area delay model has high precision.

\section{CONCLUSION}

A method of area delay modeling is proposed in this paper. Traffic flow of each path is obtained according to traffic assignment. Path delay is calculated by analyzing the rules that traffic flow runs through upstream intersection and downstream intersection. On these basis, area delay is figured out. This area delay model reflects the inherent mechanism of urban traffic area delay, and it is important for traffic flow property in area and area coordination control.

\section{REFERENCES}

[1] Hongbin Yin, Jianmin Xu, The Technology of road traffic controlling, M . South China University of Technology press, 2000.

[2] Wardrop J G. Some Theoretical Aspects of Road Traffic Research, C . 1952, pp. 325-362.

[3] Sheffi Y. Urban Transportation Networks: Equilibrium Analysis with Mathematical Programming Methods [M]. Prentice-Hall,1985.

[4] Liu H X,He X,He B. Method of Successive Weighted Averages (MSWA) and Self-Regulated Averaging Schemes for Solving Stochastic User Equilibrium Problem [J]. Networks and SpatialEconomics, 2009, 9(4), pp. $485-503$.

[5] En Wu, Xiaoguang Yang, Zhen Wu,YunTao Chang, Parameters Cooptimization for Artery Coordinated Control Based on Genetic Algorithm .J. Journal of Tongji University(Natual Science), 2008, 36(7),pp. 921-926.

[6] Yan Zhuang, Wenjia Zeng, Study of traffic delay model at signal intersection, J. Journal of Shenzhen University Science And Engneering, 2006,23(4),pp. 309-313.

[7] Xujun Wan, Huapu Lu, An Optimal offset Model for artery traffic signal control system, J. China Journal of Highway and Transport, 2001,14(2),pp. 99-102. 